\title{
All neuropathies great and small
}

\author{
Ellen B. Penny and Brian D. McCabe
}

Center for Neurobiology and Behavior and Department of Physiology and Cellular Biophysics, Columbia University College of Physicians and Surgeons, New York, New York, USA.

\begin{abstract}
Autosomal-dominant pure hereditary spastic paraplegia (AD-HSP) is characterized by the degeneration of long axons in corticospinal tracts and dorsal columns, resulting in spasticity and difficulty walking. Mutations in the SPG4 gene product spastin are the predominant genetic lesions associated with this inherited disease. In this issue, Orso et al. examine and reconcile existing Drosophila mutants of spastin and generate a new model for HSP by overexpression of a fly spastin transgene that carries a mutation prevalent in human AD-HSP (see the related article beginning on page 3026). Expression of this mutant spastin protein produces pathology in flies reminiscent of the human disease, including adult locomotion defects, in addition to causing aberrant synaptic morphology and altered microtubule stability. Both movement and synaptic defects in fly mutants were ameliorated by treatment with the microtubule-modifying agent vinblastine. The results are consistent with disease-causing mutations in human spastin producing dominant-negative proteins and confirm the usefulness of Drosophila genetic techniques to understand HSP and other neurodegenerative diseases.
\end{abstract}

Good things come in small packages, and for nearly a century the minuscule fruit fly Drosophila melanogaster has been generous to researchers, leading to the development of a genetic toolkit of unrivaled sophistication. With a rapid generation time and inexpensive culture requirements, huge numbers of Drosophila can be screened in order to find rare mutations and fully exploit the advantages of a completely sequenced genome. Genetics can be coupled with molecular and cell biology, biochemistry, electrophysiology, and so on for a comprehensive phenotypic analysis. The combination has proven powerful, yielding fundamental insights into the mechanisms of development, physiology, and neurobiology, for even though flies are small, their brains are estimated to have over 250,000 neurons, allowing relatively complex behaviors. The beauty of Drosophila is not only skin (or even cuticle) deep, however - a comparison of the fly and human genomes reveals striking similarities. For example, $77 \%$ of the approximately 1,000 genes that have been linked to specific human diseases have a readily identifiable Drosophila homolog

Nonstandard abbreviations used: AD-HSP, autosomal-dominant pure HSP; HSP, hereditary spastic paraplegia; NMJ, neuromuscular junction; RNAi, RNA interference.

Conflict of interest: The authors have declared that no conflict of interest exists.

Citation for this article: J. Clin. Invest. 115:2968-2971 (2005). doi:10.1172/JCI27003.
(1). An increasing number of researchers are taking advantage of this conservation to study the basic molecular and cell biology of disease-causing genes and search for interacting molecules using the genetics of this tractable model organism.

A number of approaches are available for examining a disease-related gene in Drosophila (Figure 1). The traditional and most reliable way is to generate a mutant for the fly homolog of the disease-associated gene. With large-scale programs underway to generate mutations in the majority of Drosophila's approximately 14,000 genes, this approach is becoming easier and more rapid (2). If a disease protein has a dominant effect, another approach is to overexpress the protein in an otherwise normal fly. Thanks to the Gal4/upstream activating sequence (Gal4/UAS) binary expression system (3), transgenic proteins can be expressed in many tissues or in just small subsets of cells. For example, eye-specific overexpression of many disease-associated proteins disrupts the organization of cells in the eye, which can easily be "scored" by microscopic examination, providing a productive in vivo platform to search for mutations that suppress the deleterious effects of disease genes (4). The latest addition to the flies' genetic arsenal is transgenic RNA interference (RNAi), which relies on the ability of double-stranded RNAs to induce the degradation of homologous mRNAs by conscripting the enzymatic machinery nor- mally utilized to protect against viruses or produce microRNAs (5). To target a gene for interference, a transgenic construct is generated with inverted regions of the gene of interest. When RNA is expressed from this transgene, it forms a hairpin that is cleaved by endogenous fly enzymes into small interfering RNAs (siRNAs). These siRNAs are used as a guide by the RNAi silencing complex (RISC) to recognize and deplete homologous mRNA sequences, knocking down protein expression of the targeted gene. Unlike in plants and Caenorbabditis elegans, the RNAi effect in Drosophila seems to be cell autonomous, allowing transcripts to be targeted only in the cells where the RNAi transgene is expressed using the Gal4/UAS system.

\section{Miniature models of hereditary spastic paraplegia}

In this issue of the JCI, Orso and colleagues utilize a combination of genetic techniques to study the neurodegenerative disorder hereditary spastic paraplegia (HSP) in a Drosophila model (6). HSPs are a heterogeneous group of disorders that vary in their pattern of inheritance, time of onset, and pathology; however, all are characterized by spasticity associated with the degeneration of spinal cord axons (7). Pure HSPs exhibit retrograde axonopathy of the terminal ends of axons in corticospinal tracts and the fasciculus gracilis and were classically thought to be uncomplicated by neurological symptoms other than spasticity, although more recent evidence suggests that neuronal dysfunction can also occur outside of the motor system (8). Eleven genetic loci, including 4 characterized genes, are associated with autosomal-dominant pure HSP (AD-HSP). All 4 of the characterized AD-HSP genes - spastin (SPAST), kinesin family member 5A (KIF5A), atlastin (also known as spastic paraplegia $3 A$, SPG3A), and heat shock protein 60 (HSP60) - have Drosophila homologs. Greater than $40 \%$ of human AD-HSPs are caused by mutations in spastin, which belongs to the AAA family of adenosine triphosphatases. Spastin is related to katanin p60, which has biochemically been shown to sever microtubules. Spastin, too, can cleave microtubules 


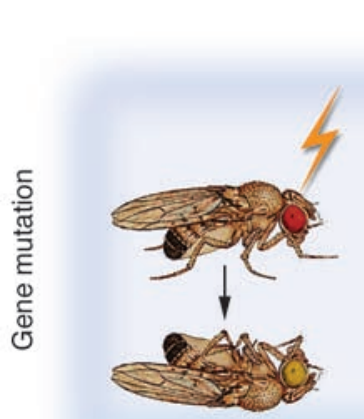

Genetic approach

Mutagenize with chemicals or transposible elements

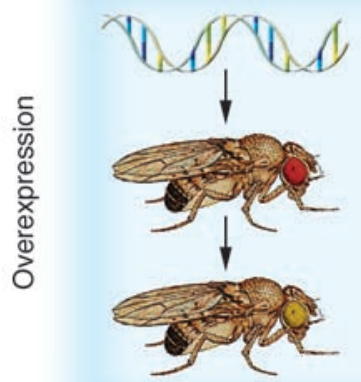

Overexpress dominant or dominant negative mutation

Screen for mutations in gene of interest as revealed by changes in phenotype or viability
Examine resulting phenotype in the whole animal or specific cells - typically the eye

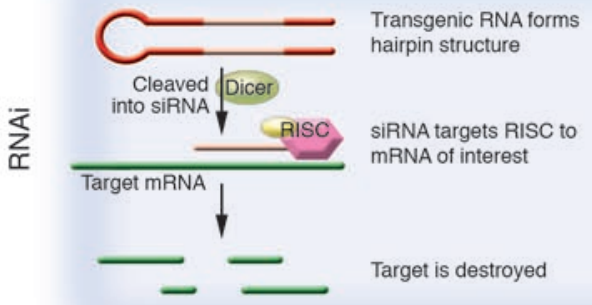

1. May overexpress human disease gene or Drosophila ortholog.

\section{May express gene in specific} spatial or temporal pattern.

3. Dominant-negative mutants may not completely inhibit endogenous protein.
1. Can generate null mutants with no gene function or hypomorphs

2. Gene function can be rescued in a tissue-specific manner to determine cell-type requirements.
1. May express RNAi in specific spatial or temporal patterns.

2. Gene expression often reduced but not completely eliminated.

3. Possibility of off-target effects?
1. HSP: mutation of spastin (12).

2. Adrenoleukodystrophy: fly gene identified by forward genetics (19)

3. Alzheimer disease: presenilin mutants (20).

4. Spinomuscular atrophy: mutation of survival motor neuron 1 (21).

1. HSP: overexpression of mutant spastin (6).

2. Polyglutamine diseases: overexpression of full-length and truncated proteins $(22,23)$.

3. Parkinson disease: overexpression of human $\alpha$-synuclein (24).

4. Alzheimer disease: overexpression of human $\beta$-amyloid precursor protein (25).

5. Tauopathies: overexpression of human Tau (16).

1. HSP: RNAi of spastin (11)

\section{Figure 1}

Currently available approaches for the examination of disease-related genes in Drosophila. RISC, RNAi silencing complex; siRNA, small interfering RNA; Dicer, a protein that produces siRNA from long, double-stranded RNAs.

when overexpressed in cell culture $(7,9,10)$. Microtubules provide structural support to axons and act as tracks along which proteins, vesicles, and other essential cargo are transported to the synapse, making them essential for the development and normal function of neurons and synaptic terminals.

\section{Suspicion of inhibition}

Last year, 2 independent groups employed different techniques to study spastin function in Drosophila $(11,12)$. Both groups determined that spastin overexpression within the nervous system was highly toxic, causing early mortality. Overexpression was associated with decreased staining of microtubules, suggesting that spastin can cleave microtubules in vivo, which has previously been demonstrated in vitro (10). However, their data conflicted concerning the effects of spastin loss of function on adult viability and neuromuscular junction
(NMJ) morphology and function. Trotta et al. (11) knocked down fly spastin expression using transgenic RNAi, while Sherwood et al. (12) generated mutations in the fly spastin gene. Approximately $20 \%$ of complete loss-of-function spastin mutants reached adulthood, with those that survived exhibiting severe motor defects, which indicates that spastin loss of function produces pathology in Drosophila reminiscent of human HSP (12). In contrast, inhibition of spastin RNA by the ubiquitous expression of a transgenic RNAi construct was reported to cause complete lethality during development (9).

Both groups also examined the requirement for spastin in the development of motor neuron synapses. The Drosophila NMJ is one of the best-characterized synapses in any organism at the morphological and physiological level. During the larval stage, Drosophila NMJ synapses grow rapidly by the addition of synaptic branches and boutons (13). Examination of NMJ morphology in spastin mutants revealed smaller, more numerous synaptic boutons at the NMJ than in control animals as well as reduced numbers of microtubule bundles (10). Electrophysiological recordings from these mutants showed that neurotransmitter release was reduced. In contrast, when spastin was inhibited in the nervous system using RNAi, the NMJ synaptic area was decreased, and staining of posttranslationally modified tubulin was increased (9). Electrophysiological examination of these RNAi-inhibited animals found increased amplitudes of excitatory junctional currents (9). No functional protein was produced by spastin mutants (10), so it was worrying that RNA interference of spastin caused an opposing phenotype. Was the transgenic RNAi technique doing something unexpected? 
Since RNAi was initially discovered, there has been speculation that problems such as lack of specificity may limit the usefulness of the technique (5). In vertebrate cell culture, RNAi has been found to cause the degradation of unrelated targets and activation of the IFN pathway $(14,15)$, though this should not be a problem in Drosophila (5). The incongruous results produced by spastin mutations versus RNAi of spastin sparked an intense discussion about the relevance and pitfalls of RNAi models in Drosophila. It was speculated that the spastin RNAi construct might have off-target activity and produce effects unrelated to spastin function. Furthermore, similar problems might arise in other RNAi models, limiting their usefulness. This was particularly disappointing because transgenic RNAi models are generally faster to produce than gene mutations and have advantages such as the ability to knock down gene expression in specific cell types or times during development, even though RNA interference suffers the disadvantage of commonly only producing partial lossof-function phenotypes (5).

\section{Concord, not conflict}

The study by Orso and colleagues in this issue of the JCI (6) has relieved some of these worries, if not resolved them. A problem with previous studies is that like was not compared with like. Whereas spastin mutations remove protein function throughout the animal, RNAi experiments had primarily characterized the effects of removing spastin expression solely in the nervous system. Orso et al. have examined the effects of interfering with spastin RNA in all tissues, not just in the nervous system. The authors demonstrate that ubiquitous spastin RNAi expression and spastinnull mutants have a similar phenotype: low eclosion rates, a decreased NMJ synaptic area with an increased number of synaptic boutons, and increased amounts of acetylated tubulin. Electrophysiological recordings were not carried out in the current study, but presumably ubiquitous RNAi expression would produce electrophysiological defects similar to those observed in spastin mutants.

Orso et al. have developed another way to model HSP, by transgenic expression of a mutant spastin protein (6). They generated a point mutation in a transgenic Drosophila spastin at a location equivalent to a prevalent mutation responsible for HSP in humans. Overexpression of this mutant protein produced pathology similar to that caused by RNAi of spastin. Whereas overexpression in the eye of wild-type spastin alone caused cellular disarray, coexpression with the mutant spastin suppressed this toxic effect. These experiments were revealing because they strongly suggest that disease-associated spastin mutations produce dominant-negative proteins that disrupt normal spastin function.

While Orso et al. (6) have provided compelling evidence that RNA interference of spastin can be effectively used to study HSP, some differences among fly spastin models still exist. In particular, how spastin loss of function affects microtubules remains unclear. Previous studies used very different methods to assess the presence of microtubules. Sherwood et al. (12) found decreased levels of $\alpha$-tubulin and the microtubule-associated protein MAP1B. Trotta et al. (11) found that although spastin RNAi increased the level of posttranslationally-modified tubulin, total tubulin staining was decreased, perhaps due to depletion of free tubulin dimers. This may account for Sherwood et al.'s finding of decreased $\alpha$-tubulin at spastin mutant synapses. Decreased MAP1B staining in spastin mutants indicates that microtubule structure may be disrupted and suggests that MAP1B may be involved in the HSP pathologic mechanism, as mutations in other microtubule-associated proteins can produce neurodegeneration (16).

\section{Alighting on new therapies}

The path is now clear to apply the full power of Drosophila genetics to elucidate the molecular and cellular mechanisms behind HSP and other neurodegenerative disorders. Several genes associated with HSP other than spastin are also required for cellular transport and trafficking. KIF5A is a neuronal kinesin superfamily member involved in fast axonal transport, while atlastin encodes a GTPase with homology to dynamins, which are associated with vesicle trafficking. Disruption of axonal transport may be a common mechanism by which mutations in these genes cause HSP and could explain why spinal neurons with long axons are particularly vulnerable to HSP. Drosophila is an ideal organism in which to study the genetic interactions among HSP genes and to decipher whether these proteins act in the same pathological pathway. Furthermore, by identifying mutations that enhance or suppress the spastin mutant phenotype, new targets for therapeutic intervention may be isolated. Orso et al. (6) show that vinblastine, a microtubule-modifying agent already used in human patients for cancer chemotherapy, ameliorates the behavioral and morphological phenotypes produced by spastin loss of function. Chemotherapy with vinblastine causes neuropathy, perhaps due to disruption of neuronal microtubules, which indicates that at clinically tolerated doses, vinblastine might have therapeutic potential for HSP (17). A number of drugs being evaluated as therapies for Huntington disease, Parkinson disease, and Alzheimer disease are effective in Drosophila models of these disorders (18), suggesting that flies may be useful for initial validation or perhaps identification of new drug treatments. If Drosophila can contribute to the development of effective therapeutic treatments for neurological diseases like HSP, more than geneticists will benefit from their gifts.

Address correspondence to: Brian D. McCabe, Department of Physiology and Cellular Biophysics and Center for Neurobiology and Behavior, Columbia University College of Physicians and Surgeons, 11-420 P\&S, 630 West 168th Street, New York, New York 10032, USA. Phone: (212) 305-3548; Fax: (212) 305-5775; E-mail: bm2157@columbia.edu.

1. Reiter, L., Potocki, L., Chien, S., Gribskov, M., and Bier, E. 2001. A systematic analysis of human disease-associated gene sequences in Drosophila melanogaster. Genome Res. 11:1114-1125.

2. Venken, K.J., and Bellen, H.J. 2005. Emerging technologies for gene manipulation in Drosophila melanogaster. Nat. Rev. Genet. 6:167-178.

3. Brand, A.H., and Perrimon, N. 1993. Targeted gene expression as a means of altering cell fates and generating dominant phenotypes. Development. 118:401-415.

4. Marsh, J.L., and Thompson, L.M. 2004. Can flies help humans treat neurodegenerative diseases? Bioessays. 26:485-496.

5. Roman, G. 2004. The genetics of Drosophila transgenics. Bioessays. 26:1243-1253.

6. Orso, G., et al. 2005. Disease-related phenotypes in a Drosophila model of hereditary spastic paraplegia are ameliorated by treatment with vinblastine. J. Clin. Invest. 115:3026-3034. doi:10.1172/JCI24694.

7. Reid, E. 2003. Science in motion: common molecular pathological themes emerge in the hereditary spastic paraplegias. J. Med. Genet. 40:81-86.

8. Fink, J.K., and Rainier, S. 2004. Hereditary spastic paraplegia: spastin phenotype and function. Arch. Neurol. 61:830-833.

9. Roll-Mecak, A., and Vale, R.D. 2005. The Drosophila homologue of the hereditary spastic paraplegia protein, spastin, severs and disassembles microtubules. Curr. Biol. 15:650-655.

10. Evans, K.J., Gomes, E.R., Reisenweber, S.M., Gundersen, G.G., and Lauring, B.P. 2005. Linking axonal degeneration to microtubule remodeling by Spastin-mediated microtubule severing. J. Cell Biol. 168:599-606.

11. Trotta, N., Orso, G., Rossetto, M.G., Daga, A., and Broadie, K. 2004. The hereditary spastic paraplegia 
gene, spastin, regulates microtubule stability to modulate synaptic structure and function. Curr Biol. 14:1135-1147.

12. Sherwood, N.T., Sun, Q., Xue, M., Zhang, B., and Zinn, K. 2004. Drosophila spastin regulates synaptic microtubule networks and is required for normal motor function. PLoS Biol. 2:e429.

13. Marques, G. 2005. Morphogens and synaptogenesis in Drosophila. J. Neurobiol. 64:417-434.

14. Jackson, A.L., et al. 2003. Expression profiling reveals off-target gene regulation by RNAi. Nat. Biotechnol. 21:635-637.

15. Sledz, C.A., Holko, M., de Veer, M.J., Silverman, R.H., and Williams, B.R. 2003. Activation of the interferon system by short-interfering RNAs. Nat. Cell Biol. 5:834-839.
16. Wittmann, C.W., et al. 2001. Tauopathy in Drosophila: neurodegeneration without neurofibrillary tangles. Science. 293:711-714.

17. Shapiro, W.R., and Young, D.F. 1984. Neurological complications of antineoplastic therapy. Acta Neurol. Scand. Suppl. 100:125-132.

18. Bonini, N.M., and Fortini, M.E. 2003. Human neurodegenerative disease modeling using Drosophila. Annu. Rev. Neurosci. 26:627-656.

19. Min, K.T., and Benzer, S. 1999. Preventing neurodegeneration in the Drosophila mutant bubblegum. Science. 284:1985-1988.

20. Ye, Y., and Fortini, M.E. 1999. Apoptotic activities of wild-type and Alzheimer's disease-related mutant presenilins in Drosophila melanogaster. J. Cell Biol. 146:1351-1364.
21. Chan, Y.B., et al. 2003. Neuromuscular defects in a Drosophila survival motor neuron gene mutant. Hum. Mol. Genet. 12:1367-1376.

22. Warrick, J.M., et al. 1998. Expanded polyglutamine protein forms nuclear inclusions and causes neural degeneration in Drosophila. Cell. 93:939-949.

23. Jackson, G.R., et al. 1998. Polyglutamine-expanded human huntingtin transgenes induce degeneration of Drosophila photoreceptor neurons. Neuron. 21:633-642.

24. Feany, M.B., and Bender, W.W. 2000. A Drosophila model of Parkinson's disease. Nature. 404:394-398.

25. Fossgreen, A., et al. 1998. Transgenic Drosophila expressing human amyloid precursor protein show gamma-secretase activity and a blistered-wing phenotype. Proc. Natl. Acad. Sci. U. S. A. 95:13703-13708.

\title{
When 7 transmembrane receptors are not G protein-coupled receptors
}

\author{
Keshava Rajagopal,1 Robert J. Lefkowitz,, ${ }^{2,3,4}$ and Howard A. Rockman',5,6 \\ ${ }^{1}$ Department of Surgery, ${ }^{2}$ Department of Medicine, ${ }^{3}$ Department of Biochemistry, ${ }^{4}$ Howard Hughes Medical Institute, \\ ${ }^{5}$ Department of Cell Biology, and ${ }^{6}$ Department of Molecular Genetics, Duke University Medical Center, Durham, North Carolina, USA.
}

\begin{abstract}
Classically, 7 transmembrane receptors transduce extracellular signals by coupling to heterotrimeric $G$ proteins, although recent in vitro studies have clearly demonstrated that they can also signal via $G$ protein-independent mechanisms. However, the physiologic consequences of this unconventional signaling, particularly in vivo, have not been explored. In this issue of the JCI, Zhai et al. demonstrate in vivo effects of G protein-independent signaling by the angiotensin II type 1 receptor (AT1R) (see the related article beginning on page 3045). In studies of the mouse heart, they compare the physiologic and biochemical consequences of transgenic cardiac-specific overexpression of a mutant AT1R incapable of $G$ protein coupling with those of a wild-type receptor. Their results not only provide the first glimpse of the physiologic effects of this newly appreciated mode of signaling but also provide important and previously unappreciated clues as to the underlying molecular mechanisms.
\end{abstract}

All vital physiologic functions of higherorder animals are critically regulated by signal transduction through 7 transmembrane receptors (7TMRs), which in the in vivo context has traditionally been understood to be mediated via heterotrimeric $G$ proteins and downstream secondmessenger molecules (1). However, for a number of years, biochemical and cellular studies have suggested that some aspects

Nonstandard abbreviations used: AT1R, angiotensin II type 1 receptor; AT1-i2m, AT1R second intracellular loop mutant; AT1-WT, WT AT1R; GRK, G protein-coupled receptor kinase; $\mathrm{Tg}$-i2 $\mathrm{m}$ mice, transgenic mice with cardiac-specific overexpression of AT1-i2m; Tg-WT mice, transgenic mice with cardiac-specific overexpression of AT1-WT; 7TMR, 7 transmembrane receptor.

Conflict of interest: The authors have declared that no conflict of interest exists.

Citation for this article: J. Clin. Invest. 115:2971-2974 (2005). doi:10.1172/JCI26950. of 7TMR-mediated signaling apparently occur independently of $\mathrm{G}$ protein activation (2). Yet, the physiologic and/or pathophysiologic roles that such novel mechanisms of signal transduction play in vivo are unknown.

Zhai et al. now provide compelling evidence for distinct physiologic consequences of $\mathrm{G}$ protein-independent signal transduction via the angiotensin II type 1 receptor (AT1R) in the heart based on their generation and study of transgenic mice with cardiac-specific overexpression of a WT AT1R (AT1-WT; Tg-WT mice) or an AT1R second intracellular loop mutant (AT1-i2m; Tg-i2m mice) (3). AT1-i2m has been shown in previous in vitro studies to be completely incapable of activating $\mathrm{G}_{\alpha} \mathrm{q}$ and $\mathrm{G}_{\alpha} \mathrm{i}$, while retaining the ability to activate molecular effectors such as Src and ERK (4). The effects of AT1-i2m overex- pression on cardiac morphology, physiology, and signal transduction were assessed and compared with those of AT1-WT.

\section{G protein-independent signal transduction via the AT1R results in ventricular hypertrophy with diminished apoptosis and a unique signaling profile} The $\mathrm{Tg}-\mathrm{i} 2 \mathrm{~m}$ mice display a pronounced cardiac phenotype, which is distinct from that of the Tg-WT mice (3). Marked ventricular dilatation and eccentric hypertrophy are present in the $\mathrm{Tg}-\mathrm{i} 2 \mathrm{~m}$ hearts to a greater extent than are observed in Tg-WT hearts. However, less cardiomyocyte apoptosis is observed in the $\mathrm{Tg}-\mathrm{i} 2 \mathrm{~m}$ hearts than in the Tg-WT hearts. These data suggest that $G$ protein-independent signal transduction via AT1-i2m results in induction of cytoprotective pathways in the heart, but that in spite of this, adverse ventricular remodeling occurs.

The $\mathrm{Tg}-\mathrm{i} 2 \mathrm{~m}$ mice also display unique electrophysiologic disturbances, as they have third-degree atrioventricular (AV) block due to impaired development of the AV node (3). Isolated cardiomyocytes from $\mathrm{Tg}-\mathrm{i} 2 \mathrm{~m}$ mice display diminished L-type $\mathrm{Ca}^{2+}$ channel currents whereas these are unimpaired in Tg-WT cells. These data point to a possible role for AT1R-mediated, $G$ protein-independent signaling in the regulation of cardiac ion channels. The authors also performed invasive hemodynamic studies that suggest a possible great- 